Annuaire suisse de politique de développement

22-2 | 2003

Société de l'information et coopération internationale

\title{
DDC : une approche centrée sur les populations
}

\section{Gerolf Weigel}

\section{OpenEdition}

\section{Journals}

Édition électronique

URL : http://journals.openedition.org/aspd/567

DOI : 10.4000/aspd.567

ISSN : 1663-9669

\section{Éditeur}

Institut de hautes études internationales et du développement

\section{Édition imprimée}

Date de publication : 1 novembre 2003

Pagination : 135-139

ISSN : 1660-5934

\section{Référence électronique}

Gerolf Weigel, « DDC : une approche centrée sur les populations », Annuaire suisse de politique de développement [En ligne], 22-2 | 2003, mis en ligne le 22 mars 2010, consulté le 08 septembre 2020. URL : http://journals.openedition.org/aspd/567 ; DOI : https://doi.org/10.4000/aspd.567 


\section{DDC: une approche centrée sur les populations}

\section{Gerolf Weigel*}

es technologies de l'information et de la communication (TIC) transforment profondément de nombreuses facettes de la vie humaine: les méthodes d'apprentissage, de travail et de communication, mais aussi les interactions entre les gouvernants et la société civile. La révolution digitale est une révolution silencieuse qui, petit à petit, façonne la société du savoir en devenir.

Alors que les TIC offrent de nouvelles opportunités à ceux qui y ont accès, d'autres en sont exclus. La fracture numérique est l'expression de profondes inégalités sociales, économiques et politiques. Une vision techniciste et étroite de la fracture numérique risque d'engendrer des actions contre-productives pouvant créer de nouvelles inégalités. La question de la production de contenus est centrale, tout spécialement de contenus locaux utiles pour le développement.

Créer des usages des TIC pour le développement est un nouveau défi qui représente un espoir pour les régions marginalisées de la planète, qui souhaitent participer et tirer bénéfice de l'économie globale de la connaissance.

Bien utilisées, les TIC sont des outils puissants pour l'émancipation, la lutte contre la pauvreté et le développement durable. L'accès aux TIC peut en effet apporter de nombreux avantages, notamment en permettant l'accès à des informations pertinentes et en ouvrant des canaux d'expression à ceux qui en sont privés. Ils peuvent ainsi mieux faire entendre leurs voix, leurs besoins, et alerter l'opinion publique. Les TIC facilitent également la mise en commun de connaissances et ouvrent de nouvelles perspectives quant aux possibilités d'échanges entre réseaux et institutions.

La DDC, en tant qu'agence leader dans le gouvernement suisse dans le domaine des «TIC pour le développement (ICT4D) ${ }^{1}$, soutient des activités et des projets, essentiellement à travers des partenariats «multi-acteurs » (c'est-à-dire qui associent des institutions publiques, privées et non lucratives) et des initiatives multilatérales. Par ailleurs, un nombre croissant de projets de la DDC ont une composante TIC. L'accent est mis sur les contenus et la construction de savoirs, ce qui va bien au-delà de la diffusion d'informations au sens strict.

Plusieurs organisations non gouvernementales et médias suisses sont partenaires de la DDC dans ce domaine. Le Sommet mondial sur la société de l'information (SMSI) est l'occasion de renforcer ces partenariats au niveau suisse, notamment

DDC, Division ICT4D (Information and Communication Technologies for Development).

1 ICT4D: Information and Communication Technologies for Development. 
grâce à la création d'une plate-forme des ONG et par la représentation des médias et des ONG dans la délégation officielle suisse.

\section{TIC et développement: I'approche DDC}

Le concept «ICT4D» a été récemment adopté par la coopération internationale. Il engendre déjà différentes politiques, qui peuvent être schématiquement regroupées en deux catégories:

$\square$ L'approche techniciste, centrée sur les technologies, est la plus répandue, notamment auprès de l'industrie, de l'Union internationale des télécommunications (UIT) et de certains programmes de la Banque mondiale. Elle repose sur le présupposé que la création d'infrastructures et d'accès aux TIC (par exemple, par la connexion d'écoles) engendre automatiquement du développement. Cette approche se concentre sur des objectifs quantitatifs (télédensité, largeur de la bande passante, etc.) et vise à faire accéder les plus pauvres au réseau mondial, bien que celui-ci soit caractérisé par une asymétrie des flux d'informations du Nord (USA et Europe essentiellement) vers le Sud. Elle repose essentiellement sur le principe de l'effet d'entraînement (trickle-down effect), sur des stratégies de promotion des investissements privés et sur l'introduction des TIC dans les administrations publiques et les services sociaux.

- L'approche centrée sur le «développement», quant à elle, conçoit les TIC comme des outils puissants, mais en aucun cas comme une fin en soi. Elle reconnaît qu'elles peuvent jouer un rôle crucial, à condition qu'elles soient intégrées dans des politiques plus larges: la communication et la construction de savoirs sont, en effet, des processus complexes, ancrés dans des environnements socio-économiques à chaque fois uniques. Cette approche reconnaît l'importance de l'accès à l'information mondiale, mais accorde une importance égale à la création et à la diffusion de contenus locaux: les pays en développement doivent pouvoir créer des contenus fondés sur leurs valeurs sociales pour traduire la diversité de l'expression culturelle. Une telle approche se fonde donc sur des priorités et des demandes locales. Elle vient compléter les approches commerciales et permet de rattacher les régions et les populations défavorisées à l'infrastructure mondiale des télécommunications.

La DDC suit clairement cette seconde approche.

\section{Les TIC, instruments de lutte contre la pauvreté}

Les TIC ne se réduisent pas à Internet et au téléphone mobile, bien que ces deux outils soient les plus visibles et les plus spectaculaires. Les populations défavorisées ont davantage accès à la radio et à la télévision. La combinaison des nouveaux moyens de communication et des médias traditionnels présente de grands potentiels, d'autant plus que les médias traditionnels, comme la radio, deviennent de plus en plus interactifs. 
Bien intégrées dans des programmes de développement, les TIC peuvent être des instruments pour lutter contre la pauvreté. Une radio locale peut, par exemple, diffuser des informations sur la santé ou l'éducation ou encore permettre d'instaurer plus de transparence dans les prises de décision politiques.

Cependant, les infrastructures ne suffisent pas et les TIC comportent aussi des risques de retours en arrière, notamment si les fonds de la coopération internationale s'orientent vers des projets trop techniques, n'ayant pas d'effets positifs pour les plus pauvres.

La DDC est convaincue que les TIC sont des instruments efficaces de lutte contre la pauvreté, principalement à travers les trois axes suivants:

- L'accès aux informations et au savoir. L'accès dépend de la présence d'infrastructures et des capacités humaines et institutionnelles à utiliser les informations. Il dépend aussi des capacités économiques des populations à pouvoir payer cet accès. Celui-ci n'est cependant pas forcément individuel; des accès collectifs s'avèrent souvent plus appropriés d'un point de vue économique, social et culturel, et il s'agit de développer davantage cette option.

$\square$ L'expression et l'autopromotion. Les TIC peuvent faciliter l'expression citoyenne, notamment pour orienter les politiques de développement et créer des modes de décision plus décentralisés. Elles permettent aussi de promouvoir la diversité socioculturelle, notamment en donnant à tous les citoyens un accès égal aux sources du savoir, à l'information et aux médias.

$\checkmark$ Communication, réseaux et échanges. Le partage des savoirs est d'une importance cruciale dans la société de l'information, notamment par sa nature décentralisée et par la vitesse des changements en cours. Les TIC facilitent la construction de partenariats, de coalitions et d'alliances nouvelles. La communication interculturelle est aussi facilitée par l'intensification des échanges entre différentes cultures.

\section{Les programmes de la DDC}

Comme on l'a dit plus haut, la DDC est au sein des instances gouvernementales suisses l'agence leader dans le domaine des TIC pour le développement. Sa Division ICT4D coordonne les programmes. Le seco (Secrétariat d'Etat à l'économie) soutient quant à lui des projets dans le domaine du commerce électronique. L'OFCOM (Office fédéral de la communication), en tant que membre de l'UIT et agence leader pour la Suisse dans l'organisation du SMSI, coopère avec la DDC dans ce domaine.

La DDC a toujours considéré que le savoir est le capital le plus important pour le développement; il l'est d'autant plus maintenant avec l'arrivée des technologies digitales. Compte tenu de son approche «développement», elle ne soutient pas des grands projets d'infrastructures ni des projets traditionnels bilatéraux. Elle s'appuie sur des réseaux existants afin de renforcer les fondements institutionnels et organisationnels et faciliter l'expression des pays du Sud - notamment des populations désavantagées - dans les réseaux mondiaux et régionaux, avec l'espoir qu'ils pourront ainsi davantage participer aux prises de décision. 
Principaux programmes et partenaires*

\begin{tabular}{|c|c|c|}
\hline Programmes/Partenaires & Implication de la DDC & Liens \\
\hline $\begin{array}{l}\text { The Global Knowledge } \\
\text { Partnership (GPK) } \\
\text { Partenariat entre des } \\
\text { institutions publiques, } \\
\text { privées et non lucratives. }\end{array}$ & $\begin{array}{l}\text { Le directeur de la DDC est } \\
\text { le président du Comité } \\
\text { exécutif du GKP. La DDC } \\
\text { est très impliquée dans la } \\
\text { définition de la stratégie } \\
\text { GKP } 2005 \text { et la mise en } \\
\text { place d'un secrétariat } \\
\text { permanent à Kuala Lumpur. }\end{array}$ & $<$ www.globalknowledge.org > \\
\hline $\begin{array}{l}\text { Sommet mondial sur la société } \\
\text { de l'information (SMSI)/ } \\
\text { Plate-forme ICT4D et } \\
\text { World Electronic Media } \\
\text { Forum (WEMF) }\end{array}$ & $\begin{array}{l}\text { La DDC lance un espace de } \\
\text { débats et d'expositions «multi- } \\
\text { acteurs» sur les questions liées } \\
\text { aux TIC et au développement. } \\
\text { Elle cofinance également le WEMF. }\end{array}$ & $<w w w . i c t-4 d . o r g>$ \\
\hline $\begin{array}{l}\text { International Institute for } \\
\text { Communication and } \\
\text { Development (IICD) }\end{array}$ & $\begin{array}{l}\text { La DDC cofinance des projets, } \\
\text { des activités et des événements, } \\
\text { spécialement au Mali et en Bolivie. }\end{array}$ & $<$ www.iicd.org $>$ \\
\hline $\begin{array}{l}\text { Building Digital Opportunities } \\
\text { (BDO) }\end{array}$ & $\begin{array}{l}\text { La DDC participe à ce réseau } \\
\text { ayant pour objectif d'inclure } \\
\text { les plus pauvres dans la société } \\
\text { de l'information, notamment en } \\
\text { faisant le lien entre les médias } \\
\text { traditionnels et les TIC. }\end{array}$ & $<w w w$.iconnect-online.org $>$ \\
\hline One World International (OWI) & $\begin{array}{l}\text { La DDC soutient la plate-forme } \\
\text { sud-asiatique de ce réseau } \\
\text { mondial pour le développement. }\end{array}$ & $<$ www.oneworld.net> \\
\hline UN ICT Task Force & $\begin{array}{l}\text { Le directeur de la DDC est } \\
\text { membre du comité. La DDC } \\
\text { apporte également un soutien } \\
\text { financier à cette plate-forme de } \\
\text { propositions lancée par I'ONU. }\end{array}$ & $<w w w$.unicttaskforce.org $>$ \\
\hline $\begin{array}{l}\text { Centre suisse des technologies } \\
\text { de l'information dans } \\
\text { I'enseignement (CTIE) }\end{array}$ & $\begin{array}{l}\text { La DDC est partenaire du CTIE } \\
\text { pour promouvoir l'éducation à } \\
\text { distance et des échanges interna- } \\
\text { tionaux entre écoles. }\end{array}$ & $<w w w . e d u c a . c h>$ \\
\hline Partenariats divers & \multicolumn{2}{|c|}{$\begin{array}{l}\text { La DDC cofinance d'autres événements et projets, notamment } \\
\text { ceux de la Commission économique pour l'Afrique (CEA), } \\
<\text { Www.uneca.org>; de l'AMARC (World Association of } \\
\text { Community Radio Broadcasters), <www.amarc.org/africa> ; } \\
\text { de Pain pour le Prochain, <www.ppp.ch> ; et de CRIS Bolivie. }\end{array}$} \\
\hline
\end{tabular}

Source: $<w w w . s d c . a d m i n . c h / i c t 4 d>$.

* Liste complète des programmes et partenaires: <www.sdc.admin.ch/ict4d>. 
Il s'agit aussi de renforcer les organisations et les réseaux locaux pour faciliter le dialogue Sud-Sud et l'échange de savoirs. La promotion, la reconnaissance, l'usage et l'échange de savoirs locaux constituent également l'une des priorités de la DDC, dont l'intervention consiste essentiellement à soutenir des initiatives multilatérales et des partenariats «multi-acteurs». En effet, la force principale des TIC est de permettre la construction de réseaux et de partenariats entre divers milieux institutionnels, des organisations de terrain aux instances gouvernementales en passant par les entreprises privées.

\section{Leçons et orientations}

Les TIC sont déjà une réalité dans les pays en voie de développement. De fait, elles créent aussi de nouvelles inégalités, entre ceux qui y ont et ceux qui n'y ont pas accès. C'est pourquoi la demande des pays en développement est très forte pour des projets adoptant une approche «développement». Les agences d'aide ne doivent pas ignorer cet appel; il leur faut orienter leurs programmes pour faire des TIC des outils de réduction de la pauvreté ${ }^{\text {. }}$

Pour que cette orientation soit efficace, il est essentiel de créer, en parallèle, une culture de partage d'expériences, afin de mieux tirer les leçons des succès et des échecs des différentes initiatives dans le domaine ICT4D. Il s'agit d'approfondir les critères de réussite, notamment pour la création de contenus et le renforcement des capacités institutionnelles et humaines. Il s'agit également de réfléchir sur les questions de coûts et de modèles économiques viables pour les plus pauvres.

2 Gerster R. and Zimmermann S., ICT for Poverty Reduction?, SDC Discussion Paper, Bern, March 2003. 